\title{
Molecular characterization of Mycobacterium intracellulare-related strains based on the sequence analysis of $h s p 65$, internal transcribed spacer and 16S rRNA genes
}

\author{
Joo-Hee Park, ${ }^{1,2} \dagger$ Tae-Sun Shim, ${ }^{3} \dagger$ Seung-Ae Lee, ${ }^{1,2}$ Hyungki Lee, ${ }^{1,2}$ \\ In-Kyung Lee, ${ }^{1,2}$ Kijeong Kim, ${ }^{4}$ Yoon-Hoh Kook ${ }^{1,2}$ and Bum-Joon Kim ${ }^{1,2}$

\begin{abstract}
${ }^{1}$ Department of Microbiology and Immunology, Cancer Research Institute, Institute of Endemic Diseases, Seoul National University Medical Research Center (SNUMRC), Seoul National University College of Medicine, Seoul 110-799, Republic of Korea

${ }^{2}$ Liver Research Institute, Seoul National University College of Medicine, Seoul 110-799, Republic of Korea

${ }^{3}$ Division of Pulmonary and Critical Care Medicine, Department of Internal Medicine, Asan Medical Center, College of Medicine, University of Ulsan, Seoul, Republic of Korea
\end{abstract} \\ ${ }^{4}$ Department of Microbiology, School of Medicine, Chung-Ang University, Seoul, Republic of Korea
}

Correspondence

Bum-Joon Kim

kbumjoon@snu.ac.kr

Received 21 March 2010

Accepted 2 June 2010
We investigated the molecular epidemiological features of 94 Mycobacterium intracellularerelated strains, isolated from Korean patients, using sequence analysis targeting 3 independent chronometer molecules, hsp65, the internal transcribed spacer 1 region and the 16S rRNA gene. By collective consideration of these three gene-based approaches, the 94 strains were divided into 5 groups (INT1, INT2, INT3, INT4 and INT5). The frequencies of genotype INT1, 2, 3, 4 and 5 in the 94 isolates were $57.4 \%$ (54), $27.7 \%$ (26), $6.4 \%$ (6), $5.3 \%$ (5) and $3.2 \%$ (3), respectively. When correlations between genotypes and clinical parameters (age, sex, radiological type and the presence of a cavity) were analysed in 78 patients with non-tuberculous mycobacteria pulmonary diseases, no relationships were observed with respect to age, sex and radiological type, but genotype and the presence of a cavity tended to be related $(P=0.051)$.

\section{INTRODUCTION}

Members of Mycobacterium avium complex (MAC) are the most frequently isolated non-tuberculous mycobacteria (NTM). Traditionally, MAC includes two species, $M$. avium and Mycobacterium intracellulare (Falkinham, 1996; Inderlied et al., 1993; Turenne et al., 2007). Recently, advances in molecular taxonomy have fuelled identification of novel species within the MAC, including Mycobacterium chimaera (Tortoli et al., 2004), Mycobacterium colombiense (Murcia et al., 2006), Mycobacterium arosiense (Bang et al., 2008), Mycobacterium vulneris (van Ingen et al., 2009),

†These authors contributed equally to this study.

Abbreviations: ITS1, internal transcribed spacer 1; MAC, Mycobacterium avium complex; NTM, non-tuberculous mycobacteria; PRA, PCR restriction analysis; SNP, single nucleotide polymorphism.

The GenBank/EMBL/DDBJ accession numbers for the hsp65, 16S rRNA and ITS1 gene sequences of strains Asan 35609 (INT1), Asan 37680 (INT2), Asan 37889 (INT3), Asan 35644 (INT4), Asan 37990 (INT5) and Asan 37845 (INT3) reported in this paper are FJ849761-FJ849778.
Mycobacterium marseillense, Mycobacterium timonense and Mycobacterium bouchedurhonense (Ben Salah et al., 2009).

Although differentiating members of the MAC is not valuable clinically, the elucidation of genetic diversity among strains may be essential for molecular epidemiological purposes (Stout et al., 2008). For example, it could explain different pathogenic potentials between these infections in AIDS patients or geographical variations between two species (Han et al., 2005). As has been found in other countries, MAC is the NTM isolated most frequently in Korea. However, uniquely, M. intracellulare has been reported to be isolated more frequently than $M$. avium in Korea (Bai et al., 1993; Koh et al., 2005, 2006; Ryoo et al., 2008).

Details on genetic separations between closely related strains of $M$. avium have been disclosed by many researchers (Alvarez et al., 2008; Semret et al., 2006; Turenne et al., 2008), and these have resulted in its differentiation into four subspecies: $M$. avium subsp. avium, $M$. avium subsp. silvaticum, M. avium subsp. paratuberculosis and 'M. avium subsp. hominissuis' (Turenne et al., 2007; Mijs et al., 2002). 
However, comparatively little work has been conducted on the separation of $M$. intracellulare-related strains (Böddinghaus et al., 1990; Schweickert et al., 2008). In addition, the isolation frequencies of $M$. intracellulare-related strains have not been analysed comprehensively to date.

Although the $h s p 65$ gene has been reported to be the most appropriate molecular target for the differentiation of MAC strains (Mun et al., 2007; Kim et al., 2005), it has also been reported that some discrepancies exist between hsp65, internal transcribed spacer 1 (ITS1) and 16S rDNA results in terms of the differentiation of MAC (Turenne et al., 2006). Therefore, polyphasic molecular approaches rather than a single molecule-based analysis are required to elucidate the accurate molecular epidemiological features of MACs.

Therefore, to characterize the molecular epidemiological features of $M$. intracellulare related strains in the present study, we analysed the partial sequences of three independent chronometer molecules, namely, $h s p 65$, ITS1 and the $16 \mathrm{~S}$ rRNA genes in $94 \mathrm{M}$. intracellulare-related isolates from different Korean patients. After genetic separation, correlations between the determined genotypes and clinical parameters were also investigated.

\section{METHODS}

Mycobacterial isolates and clinical interpretation. A total of 94 clinical isolates (from 94 subjects) of $M$. intracellulare or MAC were used in this study. These clinical isolates were collected from the Asan Medical Center (Seoul, Republic of Korea) and were identified by PCR restriction analysis (PRA) of the $r p o B$ gene (Lee et al., 2000).

NTM pulmonary diseases were diagnosed according to the guidelines issued by the American Thoracic Society (Griffith et al., 2007). In all subjects, age and sex information were gathered, and in the case of NTM pulmonary diseases, additionally radiological types (upper lobe cavitary vs nodular bronchiectatic type) and the presence of a cavity were analysed by computed tomography scans.

M. intracellulare genotypes were compared between patients with NTM pulmonary disease and subjects without NTM pulmonary disease. In cases with NTM pulmonary diseases, relationships between genotype and clinical findings (age, sex, radiological type and presence of a cavity) were also analysed.

Genetic analysis. Chromosomal DNA was extracted using the bead beater-phenol extraction method. Amplifications of the hsp 65, ITS1 and 16S rRNA genes were amplified in a final volume of $20 \mu \mathrm{l}$. For hsp65 gene analysis, amplicons of two different sizes were produced. For the genetic screening of all 94 strains, the partial 644 bp $h s p 65$ sequences (nucleotide positions 163 to 806 in the hsp65 gene of Mycobacterium tuberculosis) were amplified and $422 \mathrm{bp}$ of the amplified sequences were directly sequenced as previously described (Kim et al., 2006) (Table 1). In 20 strains representing the different genotypes, a near complete 1621 bp $h s p 65$ gene (nucleotide position 12 to 6 bases downstream of the terminator codon in the hsp65 gene of $M$. tuberculosis) was amplified using a primer system reported elsewhere (Turenne et al., 2006). Of the 1621 bp hsp65 amplicons, excluding ambiguous data, $1503 \mathrm{bp}$ sequences [nucleotide positions 79 to 1581 in the $h s p 65$ gene of M. tuberculosis (M15467)] were used for phylogenetic analysis. For ITS1 analysis, complete ITS1 sequences (280 bp) were directly sequenced using the two primers used for PCR and for the phylogenetic analysis (Frothingham \& Wilson, 1993) (Table 1). For 16S rRNA gene analysis, the partial 16S rRNA sequences (695 bp) encompassing hypervariable region A were also directly determined for phylogenetic analysis as described previously.

Sequence comparisons of the 16S rRNA, ITS1 and hsp65 genes were performed by BLAST analysis using sequences in the National Center for Biotechnology Information database (http://www.ncbi.nlm.nih. gov/) by referring to published data. Sequences were aligned using MEGALIGN in DNASTAR. Phylogeny reconstruction of all sequence alignments was performed in MEGA 4.1 using the neighbour-joining method (Kumar et al., 2008). Bootstrap values of the tree were computed by resampling 1000 times. Phylogenetic analyses were based on nearly complete $h s p 65$ sequences (1503 bp) and complete ITS1 sequences $(280 \mathrm{bp})$. Additionally, phylogenetic analysis based on the concatenation (2487 bp) of the three genes [hsp65 (1503 bp) + ITS1 (280 bp) + 16S rRNA (695 bp)] was performed.

Statistical analysis. All analyses were performed using SPSS software (version 12.0; SPSS). Groups were compared using Student's $t$-test for age, and the $\chi^{2}$ and Fisher's exact tests for categorical variables. All significance tests were two sided, and $P$ values of $<0.05$ were considered statistically significant.

Nucleotide accession numbers. Sequences of three genes [hsp65 gene (1503 bp), 16S rRNA (695 bp) and ITS1 gene (280 bp)] of a

Table 1. Gene names, primer sequences, amplicon sizes and sequence sizes for genotypic differentiation between $M$. intracellularerelated strains

\begin{tabular}{|c|c|c|c|c|c|}
\hline Gene & Primer orientation & Primer sequence ${ }^{\star}$ & Amplicon (bp) & Sequence $(b p)^{*}$ & No. of strains $\dagger$ \\
\hline \multirow[t]{4}{*}{ hsp65 } & $\mathrm{F}$ & $5^{\prime}$-ATCGCCAAGGAGATCGAGCT-3' & 644 & 422 & 94 \\
\hline & $\mathrm{R}$ & 5'-AAGGTGCCGCGGATCTTGTT-3' & & & \\
\hline & $\mathrm{F}$ & 5'-AATTGCGTACGACGAAGAGG-3' & 1621 & 1503 & 20 \\
\hline & $\mathrm{R}$ & 5'-ACGGACTCAGAAGTCCATGC-3' & & & \\
\hline \multirow[t]{2}{*}{ ITS1 } & $\mathrm{F}$ & 5'-TTGTACAACACCGCCCGTC-3' & 460 & 280 & 94 \\
\hline & $\mathrm{R}$ & 5'-TCTCGATGCCAAGGCATC-3' & & & \\
\hline \multirow{2}{*}{$16 \mathrm{~S}$ rRNA } & $\mathrm{F}$ & 5'- TTGTACAACACCGCCCGTCA-3' & 1038 & 695 & 94 \\
\hline & $\mathrm{R}$ & 5'-TCTCGATGCCAAGGCATCCACC-3' & & & \\
\hline
\end{tabular}

F, Forward; R, reverse.

${ }^{\star}$ Sequences for the phylogenetic analysis.

$\dagger$ Numbers of strains analysed by this method. 
Table 2. Clinical presentation of patients used in this study

\begin{tabular}{|c|c|c|}
\hline \multicolumn{2}{|l|}{ Clinical factor } & $\begin{array}{l}\text { No. of } \\
\text { patients }\end{array}$ \\
\hline \multicolumn{3}{|c|}{ Total patients $($ no. $=94)$} \\
\hline rpoB PRA (\%) & $\begin{array}{l}\text { Identified as } M \text {. intracellulare } \\
\text { Identified as MAC }\end{array}$ & $\begin{aligned} 93 & (98.9) \\
1 & (1.1)\end{aligned}$ \\
\hline $\begin{array}{l}\text { Age (years) } \\
\text { mean } \pm \mathrm{SD}\end{array}$ & & $61.0 \pm 13.3$ \\
\hline Male (\%) & & $40(42.6)$ \\
\hline \multirow[t]{2}{*}{ Disease $(\%)$} & Disease present & $78(83.0)$ \\
\hline & Disease absent & $16(17.0)$ \\
\hline \multicolumn{3}{|c|}{ Patients with disease $($ no. $=78)$} \\
\hline rpoB PRA & $\begin{array}{l}\text { Identified as } M \text {. intracellulare } \\
\text { Identified as MAC }\end{array}$ & $\begin{aligned} 77 & (98.7) \\
1 & (1.3)\end{aligned}$ \\
\hline $\begin{array}{c}\text { Age (years), } \\
\text { mean } \pm \mathrm{SD}\end{array}$ & & $61.3 \pm 12.4$ \\
\hline Male (\%) & & $33(42.3)$ \\
\hline \multirow[t]{3}{*}{ CXR type $\neq$} & Upper lobe cavitary type & $27(34.6)$ \\
\hline & Nodular bronchiectatic type & $46(59.0)$ \\
\hline & $\begin{array}{l}\text { Not belonging to these two } \\
\text { radiological types }\end{array}$ & $5(6.4)$ \\
\hline \multirow[t]{2}{*}{ Cavity§ } & Present & $29(37.2)$ \\
\hline & Absent & $49(62.8)$ \\
\hline
\end{tabular}

total of six strains, including five representing the different groups [INT1 - Asan 35609, INT 2 - Asan 37680, INT 3 - Asan 37889, INT 4 - Asan 35644 and INT 5 - Asan 37990] and one strain [INT 3 - Asan 37845] that differed slightly from INT 3 were deposited in GenBank under accession numbers FJ849761 to FJ849778.

\section{RESULTS AND DISCUSSION}

\section{Clinical data of patients}

The mean age of the 94 subjects was $61.0 \pm 13.3$ years, and the male to female ratio was $40: 54$. Ninety-three clinical isolates were identified as $M$. intracellulare by PRA assay of the $r p o B$ gene and the other isolate was identified as
MAC. Seventy-eight subjects were diagnosed as having NTM pulmonary diseases according to American Thoracic Society guidelines (Griffith et al., 2007). The mean age of the 78 patients with NTM pulmonary disease was $61.3 \pm 12.4$ years old, and the male to female ratio was 33:45. The patients with and without NTM pulmonary disease had similar ages and male to female ratios. A total of 27 (34.6\%) had upper lobe cavitary type disease based on radiological findings, 46 (59.0\%) had the nodular bronchiectatic type, and the remaining 5 $(6.4 \%)$ did not belong to either of these two radiological types (Table 2).

\section{Genotyping of $M$. intracellulare-related strains by hsp65 analysis}

Based on 422 bp hsp65 sequence analysis findings, the 94 $M$. intracellulare-related isolates were divided into four different sequevars (Table 3). According to isolated frequencies, patients were allocated to the following four sequevar groups: HG1 (63.8\%), HG2 (27.7\%), HG3 $(5.3 \%)$ and HG4 (3.2\%) (Table 3). Phylogenetic analysis based on determined hsp65 sequences (1503 bp) also showed that 94 strains could be clearly divided into four distinct groups (Fig. 1a). HG1 isolates (the most prevalent) had an $h s p 65$ sequence identical to that of $M$. chimaera type strain (DSM $44623^{\mathrm{T}}$ ). HG2 isolates (the second most prevalent) had an $h s p 65$ sequence identical to that of $M$. intracellulare type strains (ATCC $13950^{\mathrm{T}}$ ), whereas HG3 isolates had hsp65 sequences that were closely related to $M$. intracellulare, with only a $2 \mathrm{bp}$ mismatch among 1503 bp sequences. On the other hand, HG4, the most phylogenetically unique of the Korean isolates, had unique hsp65 sequences, which differed substantially from BLAST sequences, that is, a 10 and a $18 \mathrm{bp}$ mismatch were found in the $1503 \mathrm{bp} h s p 65$ sequence between this isolate and $M$. intracellulare type strain, and between the isolate and M. chimaera type strain, respectively.

Table 3. Genotype determination of $94 \mathrm{M}$. intracellulare-related strains from Korean patients by three independent molecular approaches

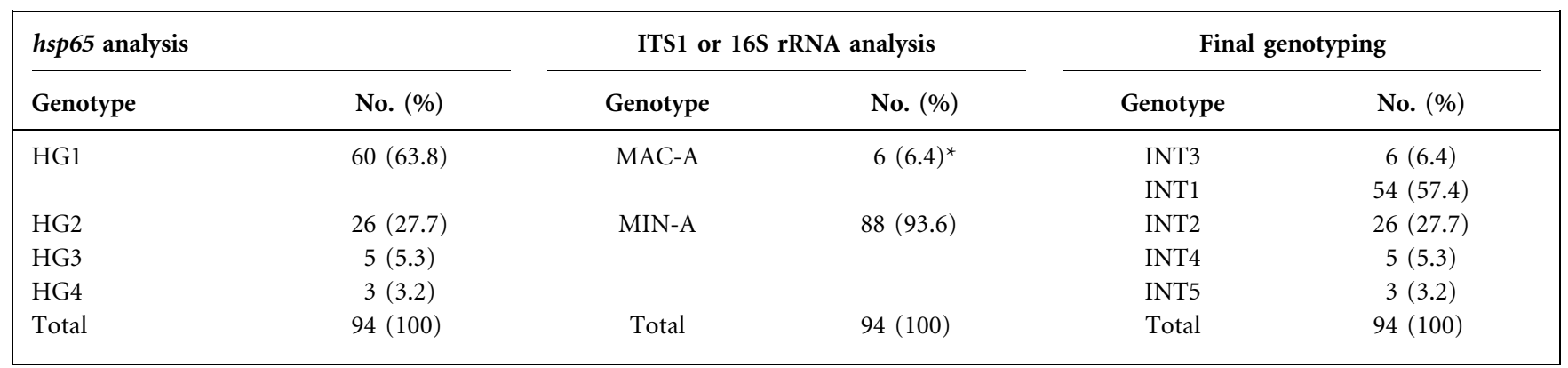

${ }^{\star}$ Five strains of six with ITS1 sequences of MAC-A type showed 16S rRNA sequences identical to M. chimaera, the other one had sequences identical to $M$. intracellulare. 

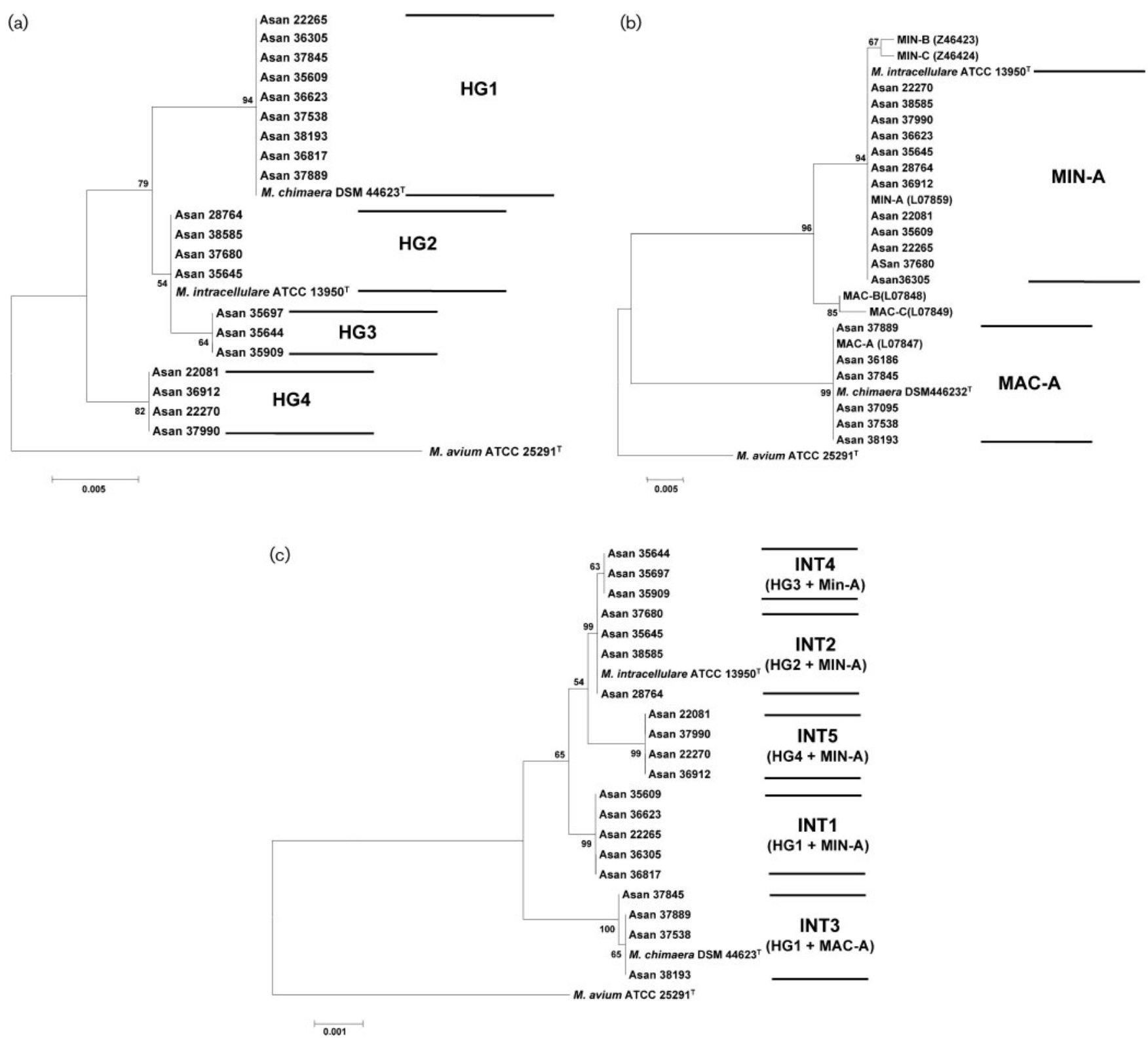

Fig. 1. M. intracellulare-related strains from Korean patients were divided into four genotypes (HG1 to HG4), two genotypes (MIN-A and MAC-A) and five genotypes (INT1 to INT5) by phylogenetic analysis based on (a) nearly complete hsp65 (1503 bp) sequence, (b) complete ITS1 (280 bp) sequence and (c) concatenation (2478 bp) of three independent genes (hsp65, ITS1 and 16S rRNA genes), respectively. Trees were constructed using the neighbour-joining method. The percentages indicated at nodes represent bootstrap levels supported by 1000 resampled datasets. Bootstrap values of $<50 \%$ are not shown. In all three phylogenetic analyses, $M$. avium was used as the outgroup species. The numbers in parentheses indicate the GenBank accession numbers. Bars, differences in nucleotide sequences.

\section{Genotyping by ITS1}

ITS1 sequence analysis divided the 94 isolates into 2 sequevars. In the present study, ITS1 sequevars were named as reported by Stout et al. (2008). Most isolates (95.7\%) belonged to MIN-A, in which the ITS1 sequence was identical to that of the $M$. intracellulare type strain. The remaining six isolates $(4.3 \%)$ belonged to MAC-A, in which the ITS1 sequence was identical to that of the $M$. chimaera type strain. All isolates belonging to HG2, HG3 and HG4 by $h s p 65$ analysis had ITS1 sequences identical to MIN-A, and only 6 of 60 strains belonging to HG1 showed MAC-A sequences identical to ITS1 of M. chimaera.

ITS1 results showed that most of our Korean isolates belonged to the MIN-A group (86/94, $91.5 \%$ ), which is not in agreement with a German study (Schweickert et al., 2008) that found that most isolates belong to MAC-A (143/ 
Table 4. Distribution of patients with or without NTM disease between the different genotypes

\begin{tabular}{|lccc|}
\hline Genotype $^{*}$ & $\begin{array}{c}\text { No. of patients (\%) } \\
\text { with NTM disease }\end{array}$ & $\begin{array}{c}\text { No. of patients (\%) } \\
\text { with no disease }\end{array}$ & Total no. (\%) \\
\hline INT1 & $44(56.4)$ & $10(62.5)$ & $54(57.4)$ \\
INT2 & $24(30.8)$ & $2(12.5)$ & $26(27.7)$ \\
INT3 & $3(3.8)$ & $3(18.8)$ & $6(6.4)$ \\
INT4 & $4(5.1)$ & $1(6.3)$ & $5(5.3)$ \\
INT5 & $3(3.8)$ & $0(0)$ & $3(3.2)$ \\
Total & $78(100)$ & $16(100)$ & $94(100)$ \\
\hline
\end{tabular}

${ }^{\star}$ The frequencies of genotypes were not significantly different between the patients with or without NTM pulmonary diseases.

166, 86.1\%), M. chimaera sp. nov. The remarkable difference shown between the frequencies of strains isolated in the two regions might reflect differences in environmental, clinical or epidemiological factors.

\section{Genotyping by 165 rRNA gene analysis}

The results of $16 \mathrm{~S}$ rRNA gene analysis were almost identical to those obtained by ITS1 analysis, with the exception of one isolate (Asan 37845). It has been reported that only one single nucleotide polymorphism (SNP) at nucleotide position 403 in the $16 \mathrm{~S}$ rRNA sequence separates $M$. chimaera and M. intracellulare (Tortoli et al., 2004). All 88 isolates identified as $M$. intracellulare (MIN-A) by ITS1 analysis also had 16S rRNA sequences identical to $M$. intracellulare. Five of the six isolates identified as $M$. chimaera by hsp65 (HG1) and ITS1 analysis (MAC-A) also had a $16 \mathrm{~S}$ rRNA SNP unique to $M$. chimaera, but interestingly, the Asan 37845 isolate had an SNP unique to M. intracellulare.

\section{Genotyping using the findings of the three sets of genetic analyses}

Taking into consideration all three analyses, the $M$. intracellulare-related strains were divided into five major groups. Phylogenetic analysis based on concatenation (2478 bp) of the three genes ( $h s p 65$, ITS1 and 16S rRNA gene) supported these groupings (Fig. 1c). These groups were named INT1 to INT5, respectively: INT1 had a $M$. chimaera identical hsp65 genotype (HG1), but $M$. intracellulare identical ITS1 (MIN-A) and 16S rRNA genotypes; INT2 had $M$. intracellulare identical sequences in all three genes (HG2 and MIN-A). INT3 had a M. chimaera identical genotype in all three genes; INT4 had a $M$. intracellulare closely related hsp65 genotype (HG3) and identical ITS1 (MIN-A) and 16S rRNA genotypes; finally, INT5 had a unique $h s p 65$ genotype specific to Korean patients (HG4), but M. intracellulare identical ITS1 (MIN-A) and 16S rRNA genotypes (Table 3, Fig. 1c). The proportions of the five groups INT1-INT5 among the 94 isolates were 57.4, 27.7, 6.4, 5.3 and $3.2 \%$, respectively (Table 3 ).

In this study, hsp65 analysis divided all 94 strains into 4 sequevars, and ITS1 or 16S rRNA gene analysis divided them into only 2 sequevars. This result strongly supports the conclusion of a previous report that hsp65 analysis has a greater ability to resolve MAC strains (Turenne et al., 2006). Furthermore, the two hsp65 sequevars (HG-3 and HG-4) were found to possess unique sequences that did not perfectly match previous database entries by BLAST analysis. In particular, HG-4 strains that formed the most distantly related phylogroup by $h s p 65$ gene analysis appear to represent a new species.

INT1, the most prevalent in Korea, had a fused genotype with a $M$. chimaera identical hsp65 sequence, and $M$. intracellulare identical ITS1 and 16S rRNA gene sequences. Given that this genotype has not been mentioned in any

Table 5. Distribution of patients with or without cavities between the genotypes

\begin{tabular}{|lcccccc|}
\hline \multirow{2}{*}{ Cavity } & \multicolumn{9}{c}{ No. (\%) of patients with: } & \multirow{2}{*}{ Total no. (\%) } \\
\cline { 2 - 6 } & INT1 & INT2 & INT3 & INT4 & INT5 & \\
\hline+ & $18(40.9)$ & $5(20.8)$ & $2(66.7)$ & $1(25)$ & $3(100)$ & $29(37.2)$ \\
- & $26(59.1)$ & $19(79.2)$ & $1(33.3)$ & $3(75)$ & $0(0.0)$ & $49(62.8)$ \\
Total & $44(100)$ & $24(100)$ & $3(100)$ & $4(100)$ & $3(100)$ & $78(100)$ \\
\hline
\end{tabular}

${ }^{*}$ The correlation between genotype and cavity is shown $(P=0.051)$. Symbols indicate the presence $(+)$ or absence $(-)$ of a cavity. 
report to date, we speculate that it might be unique to Korean patients. This also raises the possibility that INT1 strains might represent an evolutionary transitional state between M. intracellulare (INT2) and M. chimaera (INT3).

\section{Relationships between clinical data and genotypes}

The frequencies of genotypes were not significantly different between patients with and without NTM diseases (Table 4). When the relationships between genotypes and clinical parameters (age, sex, radiological types and presence of a cavity) were analysed in 78 patients with NTM pulmonary diseases, no significant relationships were observed (data not shown). However, a borderline relationship between genotypes and cavity formation was found $(P=0.051)$ (Table 5). The presence of a pulmonary cavity is a characteristic finding of mycobacterial lung disease. In the case of MAC disease, cavitation is considered as a marker of severe disease compared with less severe non-cavitary disease. According to American Thoracic Society guidelines, inclusion of an injectable drug in addition to oral drugs is recommended for cavitary MAC disease (Griffith et al., 2007).

In a published study, it was suggested that M. chimaera sp. nov. (MAC-A ITS1 group) is less virulent than $M$. intracellulare (MIN-A ITS1 group) (Schweickert et al., 2008). Our results also showed that INT1 $(78.6 \%)$ and INT2 $(92.3 \%)$ accounted for higher proportions of disease than the INT3 group (50\%), although these differences were not statistically significant (Table 4).

Furthermore, our data show relationships between genotypes and cavity formation, which suggests that the five groups have different underlying pathogenic mechanisms. In particular, relatively higher percentages had a cavity in the two groups taxonomically most distant from $M$. intracellulare, that is, INT3 (66.7\%) and INT5 (100\%) (Table 5). The differences between these genotypes in terms of pathogenic potentials and epidemiological features remain to be proved by future studies.

\section{Conclusion}

In conclusion, a total of $94 \mathrm{M}$. intracellulare-related strains isolated from Korean patients were divided into five different genotypes, based on combined genetic analysis targeting three genes, namely, $h s p 65$, ITS1 and $16 \mathrm{~S}$ rDNA. The data based on ITS1 sequence analysis showed the high homogeneity of Korean strains of $M$. intracellulare, which belonged to only the MIN-A group. We believe that this study provides novel insight into the genetic diversity of $M$. intracellulare-related strains.

\section{ACKNOWLEDGEMENTS}

This study was supported by a grant from the Korea Healthcare Technology R and D Project, Ministry of Health, Welfare and Family Affairs, Republic of Korea (A091121).

\section{REFERENCES}

Alvarez, J., García, I. G., Aranaz, A., Bezos, J., Romero, B., de Juan, L., Mateos, A., Gómez-Mampaso, E. \& Domínguez, L. (2008). Genetic diversity of Mycobacterium avium isolates recovered from clinical samples and from the environment: molecular characterization for diagnostic purposes. J Clin Microbiol 46, 1246-1251.

Bai, G. H., Park, K. S. \& Kim, S. J. (1993). Clinically isolated mycobacteria other than Mycobacterium tuberculosis from 1980 to 1990 in Korea. J Korean Soc Microbiol 28, 1-5.

Bang, D., Herlin, T., Stegger, M., Andersen, A. B., Torkko, P., Tortoli, E. \& Thomsen, V. O. (2008). Mycobacterium arosiense sp. nov., a slowly growing, scotochromogenic species causing osteomyelitis in an immunocompromised child. Int J Syst Evol Microbiol 58, 2398-2402.

Ben Salah, I., Cayrou, C., Raoult, D. \& Drancourt, M. (2009). Mycobacterium marseillense sp. nov., Mycobacterium timonense sp. nov. and Mycobacterium bouchedurhonense sp. nov., members of the Mycobacterium avium complex. Int J Syst Evol Microbiol 59, 28032808.

Böddinghaus, B., Wolters, J., Heikens, W. \& Böttger, E. C. (1990). Phylogenetic analysis and identification of different serovars of Mycobacterium intracellulare at the molecular level. FEMS Microbiol Lett 58, 197-203.

Falkinham, J. O. (1996). Epidemiology of nontuberculous mycobacteria. Clin Microbiol Rev 9, 177-215.

Frothingham, R. \& Wilson, K. H. (1993). Sequence-based differentiation of strains in the Mycobacterium avium complex. J Bacteriol 175, 2818-2825.

Griffith, D. E., Aksamit, T., Brown-Elliott, B. A., Catanzaro, A., Daley, C., Gordin, F., Holland, S. M., Horsburgh, R., Huitt, G. \& other authors (2007). An official ATS/IDSA statement: diagnosis, treatment, and prevention of nontuberculous mycobacterial diseases. Am J Respir Crit Care Med 175, 367-416.

Han, X. Y., Tarrand, J. J., Infante, R., Jacobson, K. L. \& Truong, M. (2005). Clinical significance and epidemiologic analyses of Mycobacterium avium and Mycobacterium intracellulare among patients without AIDS. J Clin Microbiol 43, 4407-4412.

Inderlied, C. B., Kemper, C. A. \& Bermudez, L. E. (1993). The Mycobacterium avium complex. Clin Microbiol Rev 6, 266-310.

Kim, H., Kim, S. H., Shim, T. S., Kim, M. N., Bai, G. H., Park, Y. G., Lee, S. H., Cha, C. Y., Kook, Y. H. \& Kim, B. J. (2005). PCR restriction fragment length polymorphism analysis (PRA)-algorithm targeting $644 \mathrm{bp}$ heat shock protein 65 ( $h s p 65)$ gene for differentiation of Mycobacterium spp. J Microbiol Methods 62, 199-209.

Kim, H. J., Mun, H. S., Kim, H., Oh, E. J., Ha, Y., Bai, G. H., Park, Y. G., Cha, C. Y., Kook, Y. H. \& Kim, B. J. (2006). Differentiation of mycobacterial species by hsp65 duplex PCR followed by duplex-PCRbased restriction analysis and direct sequencing. J Clin Microbiol 44, 3855-3862.

Koh, W. J., Kwon, O. J. \& Lee, K. S. (2005). Diagnosis and treatment of nontuberculous mycobacterial pulmonary diseases: a Korean perspective. J Korean Med Sci 20, 913-925.

Koh, W. J., Kwon, O. J., Jeon, K., Kim, T. S., Lee, K. S., Park, Y. K. \& Bai, G. H. (2006). Clinical significance of nontuberculous mycobacteria isolated from respiratory specimens in Korea. Chest 129, 341348.

Kumar, S., Dudley, J., Nei, M. \& Tamura, K. (2008). MEGA: a biologistcentric software for evolutionary analysis of DNA and protein sequences. Brief Bioinform 9, 299-306.

Lee, H., Park, H. J., Cho, S. N., Bai, G. H. \& Kim, S. J. (2000). Species identification of mycobacteria by PCR-restriction fragment length polymorphism of the rpoB gene. J Clin Microbiol 38, 2966-2971. 
Mijs, W., de Haas, P., Rossau, R., Van der Laan, T., Rigouts, L., Portaels, F. \& van Soolingen, D. (2002). Molecular evidence to support a proposal to reserve the designation Mycobacterium avium subsp. avium for bird-type isolates and ' $M$. avium subsp. hominissuis' for the human/porcine type of M. avium. Int J Syst Evol Microbiol 52, $1505-1518$.

Mun, H. S., Kim, H. J., Oh, E. J., Kim, H., Park, Y. G., Bai, G. H., Do, J., Cha, C. Y., Kook, Y. H. \& Kim, B. J. (2007). Direct application of AvaII PCR restriction fragment length polymorphism analysis (AvaII PRA) targeting $644 \mathrm{bp}$ heat shock protein 65 (hsp65) gene to sputum samples. Microbiol Immunol 51, 105-110.

Murcia, M. I., Tortoli, E., Menendez, M. C., Palenque, E. \& Garcia, M. J. (2006). Mycobacterium colombiense sp. nov., a novel member of the Mycobacterium avium complex and description of MAC-X as a new ITS genetic variant. Int J Syst Evol Microbiol 56, 2049-2054.

Ryoo, S. W., Shin, S., Shim, M. S., Park, Y. S., Lew, W. J., Park, S. N., Park, Y. K. \& Kang, S. (2008). Spread of nontuberculous mycobacteria from 1993 to 2006 in Koreans. J Clin Lab Anal 22, 415-420.

Schweickert, B., Goldenberg, O., Richter, E., Göbel, U. B., Petrich, A., Buchholz, P. \& Moter, A. (2008). Occurrence and clinical relevance of Mycobacterium chimaera sp. nov., Germany. Emerg Infect Dis 14, 1443-1446.

Semret, M., Turenne, C. Y., de Haas, P., Collins, D. M. \& Behr, M. A. (2006). Differentiating host-associated variants of Mycobacterium avium by PCR for detection of large sequence polymorphisms. J Clin Microbiol 44, 881-887.

Stout, J. E., Hopkins, G. W., McDonald, J. R., Quinn, A., Hamilton, C. D., Reller, L. B. \& Frothingham, R. (2008). Association between 16S-23S internal transcribed spacer sequence groups of Mycobacterium avium complex and pulmonary disease. J Clin Microbiol 46, 2790-2793.

Tortoli, E., Rindi, L., Garcia, M. J., Chiaradonna, P., Dei, R., Garzelli, C., Kroppenstedt, R. M., Lari, N., Mattei, R. \& other authors (2004). Proposal to elevate the genetic variant MAC-A, included in the Mycobacterium avium complex, to species rank as Mycobacterium chimaera sp. nov. Int J Syst Evol Microbiol 54, 1277-1285.

Turenne, C. Y., Semret, M., Cousins, D. V., Collins, D. M. \& Behr, M. A. (2006). Sequencing of hsp65 distinguishes among subsets of the Mycobacterium avium complex. J Clin Microbiol 44, 433-440.

Turenne, C. Y., Wallace, R., Jr \& Behr, M. A. (2007). Mycobacterium avium in the postgenomic era. Clin Microbiol Rev 20, 205-229.

Turenne, C. Y., Collins, D. M., Alexander, D. C. \& Behr, M. A. (2008). Mycobacterium avium subsp. paratuberculosis and M. avium subsp. avium are independently evolved pathogenic clones of a much broader group of M. avium organisms. J Bacteriol 190, 2479-2487.

van Ingen, J., Boeree, M. J., Kösters, K., Wieland, A., Tortoli, E., Dekhuijzen, P. N. \& van Soolingen, D. (2009). Proposal to elevate Mycobacterium avium complex ITS sequevar MAC-Q to Mycobacterium vulneris sp. nov. Int J Syst Evol Microbiol 59, 2277-2282. 